\title{
VIRGO sensitivity to binary coalescences and the Population III black hole binaries
}

\author{
K. Kulczycki ${ }^{1}$, T. Bulik ${ }^{2,1}$, K. Belczyński ${ }^{3,4}$, and B. Rudak ${ }^{1}$ \\ 1 Nicolaus Copernicus Astronomical Center, Bartycka 18, 00716 Warsaw, Poland \\ e-mail: bulik@camk.edu.pl \\ 2 Astronomical Observatory, Warsaw University, Al Ujazdowskie 4, 00478 Poland \\ 3 New Mexico State University, Department of Astronomy, 1320 Frenger Mall, Las Cruces, NM 88003, USA \\ 4 Tombaugh Fellow
}

Received 24 February 2006 / Accepted 4 August 2006

ABSTRACT

\begin{abstract}
Aims. We analyze the properties of VIRGO detector with the aim of studying its ability to search for coalescing black hole binaries. We focus on the remnants of the Population III stars, which currently should be massive black holes $\left(\sim 100-1000 M_{\odot}\right)$, some of them bound in binary systems. The coalescence of such binaries due to emission of gravitational waves may be currently observable. Methods. We use a binary population synthesis to model the evolution of Population III binaries.

Results. We calculate the signal to noise ratios of gravitational waves emitted by the system in each of the coalescence phase: inspiral, merger and ringdown, and provide simple formulae for the signal to noise ratio as a function of masses of the binaries. We estimate the detection rates for the VIRGO interferometer and also compare them with the estimates for the current LIGO. We show that these expected rates are similar to, or larger than the expected rates from coalescences of Population I and II compact object binaries.
\end{abstract}

Key words. gravitationals waves - stars: binaries: general

\section{Introduction}

The high frequency gravitational wave detectors are approaching their specified sensitivities. Both the LIGO observatory (Abramovici et al. 1992) and VIRGO (Bradaschia 1990) are already taking data. It is therefore very important to study in detail the detectability of different types of gravitational wave sources with the current detectors.

A new class of sources - black hole binaries originating in Population III stars - was recently suggested (Belczynski et al. 2004). Baraffe et al. (2001) have shown that zero metallicity star are stable, and can form intermediate mass $\approx 100-500 M_{\odot}$ black holes (Heger \& Woosley 2002). They have shown that the stars with the initial masses between 140 and $260 M_{\odot}$ undergo violent pair instability explosions and leave no remnant while the stars outside these mass range leave black holes with essentially the same mass as the initial star. Numerical investigation of Bromm et al. (1999, 2002) of Population III star formation indicate that their initial mass function allowed a much larger number of massive stars than that of Population I. Wyithe \& Loeb (2004) have considered detectability of gravitational waves emitted in the process of black hole growth on the Population III black hole seeds.

It is therefore important to ask a question whether Population III stars could form binaries. It was recently shown by Saigo et al. (2004) that a rotating metal free cloud may lead to formation of a metal free binary. Moreover, massive stars may form from clouds of slightly enhanced metallicity which may cool off more efficiently. All known stellar populations contain a large fraction of binaries.

The detectability analysis of Population III black hole binaries was performed by Belczynski et al. (2004) for the case of the advanced LIGO detector. In that paper a simple model of the evolution of the Population III stars was developed. The model was based on numerical calculation of stellar evolution. A top heavy initial mass function was assumed, and a binary fraction of Population III stars of $10 \%$. The number of stars was estimated with the assumption that a fraction of $10^{-3}$ of the baryonic mass in the Universe was processed in Population III binaries.

In this paper we investigate the detectability of the Population III black hole binaries with the current configuration of LIGO, and with VIRGO at the expected sensitivity. The signal to noise from a compact object coalescence for the LIGO detector has been calculated by Flanagan \& Hughes (1998). In Sect. 2 we estimate the signal to noise for VIRGO using the same formalism. The detection rate is calculated in Sect. 3, while Sect. 4 contains conclusions and discussion.

\section{Signal to noise ratio}

To calculate the signal to noise ratio of gravitational wave from binary black hole coalescence by the VIRGO detector we follow the formalism of Flanagan \& Hughes (1998). We find the average of the squared signal to noise ratio (SNR) over all orientations of and directions to the source, $<\rho^{2}>$ :

$$
\left(\frac{S}{N}\right)^{2}=<\rho^{2}>=\frac{2(1+z)^{2}}{5 \pi^{2} D(z)^{2}} \int_{0}^{\infty} \frac{1}{f^{2} S_{\mathrm{h}}(f)} \frac{\mathrm{d} E_{\mathrm{e}}}{\mathrm{d} f_{\mathrm{e}}}[(1+z) f] \mathrm{d} f
$$

where $z$ is the redshift of the system, $D(z)$ is the luminosity distance, $S_{\mathrm{h}}(f)$ is the one sided power spectral density of the interferometer and $\mathrm{d} E_{\mathrm{e}} / \mathrm{d} f_{\mathrm{e}}$ is the locally measured wave energy spectrum. We also introduce the frequency $f$ at the source and 


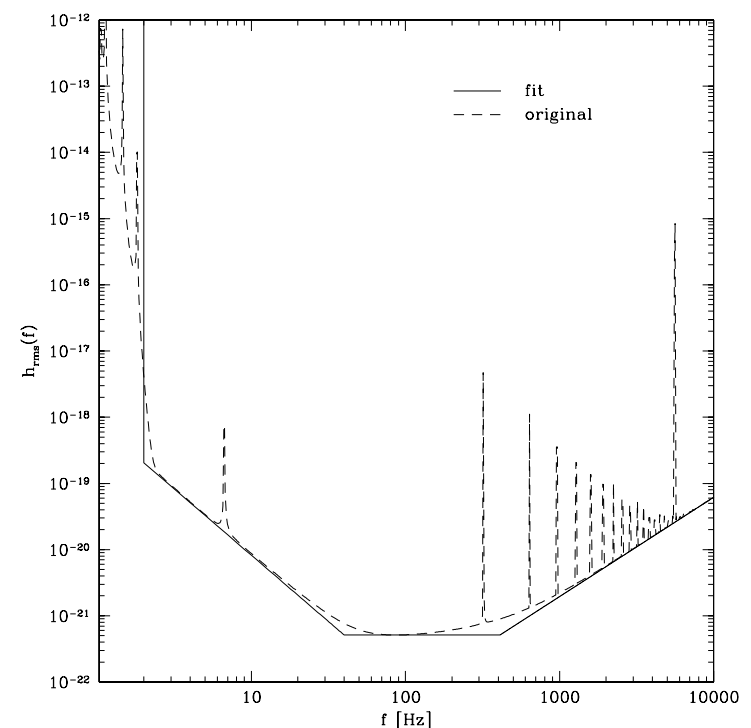

Fig. 1. The VIRGO sensitivity curve expressed as $h_{\mathrm{rms}}(f)=\sqrt{f S_{\mathrm{h}}(f)}$ (dashed lines) and the approximation used in this work (solid lines).

$f_{\mathrm{e}}=(1+z)^{-1} f$ at the interferometer. In this section we follow the system of units in which $G=c=1$.

Flanagan \& Hughes (1998) approximated the sensitivity curve of the detector with the quantity $h_{\mathrm{rms}}(f)=\sqrt{f S_{\mathrm{h}}(f)}$, i.e. root mean squared fluctuation in the noise at frequency $f$ in a bandwidth $\Delta f=f$, and then fitting them with power laws. We have analogously approximated the VIRGO sensitivity (Punturo 2004) with a simple analytical model:

$h_{\mathrm{rms}}(f)= \begin{cases}\infty & \text { for } f<f_{\mathrm{s}} \\ h_{\mathrm{m}}\left(\frac{\alpha f}{f_{\mathrm{m}}}\right)^{-2} & \text { for } f_{\mathrm{s}} \leq f<\frac{f_{\mathrm{m}}}{\alpha} \\ h_{\mathrm{m}} & \text { for } \frac{f_{\mathrm{m}}}{\alpha} \leq f \leq \alpha f_{\mathrm{m}} \\ h_{\mathrm{m}}\left(\frac{f}{\alpha f_{\mathrm{m}}}\right)^{3 / 2} & \text { for } \alpha f_{\mathrm{m}}<f\end{cases}$

with parameters:

$$
\left\{\begin{array}{l}
f_{\mathrm{s}}=2 \mathrm{~Hz} \\
f_{\mathrm{m}}=128 \mathrm{~Hz} \\
\alpha=3.2 \\
h_{\mathrm{m}}=5.12 \times 10^{-22} .
\end{array}\right.
$$

The VIRGO design sensitivity curve and the fit are shown in Fig. 1. Our approximation of the VIRGO sensitivity curve differs from the one used for the initial LIGO detector by Flanagan \& Hughes (1998) in the low frequency domain. In this domain initial LIGO sensitivity curve has been approximated by a function $h_{\text {rms }}(f) \sim f^{-3 / 2}$, while the slope of the VIRGO sensitivity curve is steeper, with $h_{\mathrm{rms}}(f) \sim f^{-2}$, yet it extends further to the low frequency regime. This modifies the analytical formulae for the signal to noise ratio, especially for the high mass merging compact objects.

The energy spectrum $\mathrm{d} E_{\mathrm{e}}[(1+z) f] / \mathrm{d} f_{\mathrm{e}}$ has different form depending on the coalescence phase: inspiral, merger or ringdown. In the following we have the same definitions of the energy spectra in these phases as used by Flanagan \& Hughes (1998). For the inspiral phase we have used the formula:

$\frac{\mathrm{d} E}{\mathrm{~d} f}=\frac{1}{3} \pi^{2 / 3} \mu M^{2 / 3} f^{-1 / 3}$,

where $\mu$ is the reduced mass of the system, while $M$ is its total mass, and $f<f_{\text {merge }}=0.02 / M$, the frequency at which the

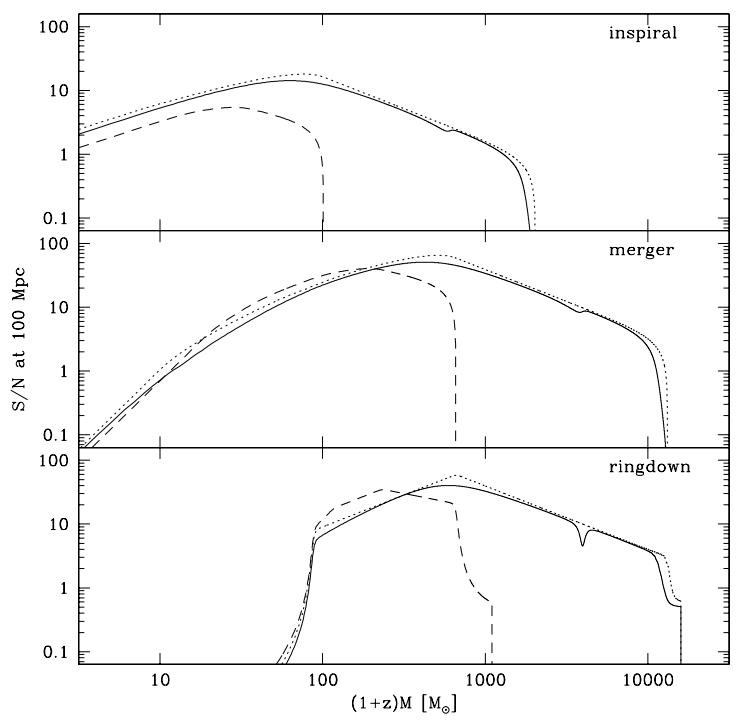

Fig. 2. SNR as a function of the redshifted total mass of a merging binary for VIRGO (solid lines) and initial LIGO (dashed lines). The dotted lines present the numerical results obtained using the approximate noise curve. The upper panel shows SNR in the inspiral phase, the middle panel in the merger phase, and the lower panel in the ringdown phase.

inspiral phase ends. The merger phase energy spectrum was approximated by a flat spectrum between $f_{\text {merge }}$ and the quasi normal mode frequency $f_{\mathrm{qnr}}=0.13 / M$ :

$$
\frac{\mathrm{d} E}{\mathrm{~d} f}=\frac{\epsilon_{\mathrm{m}} M F(\mu / M)}{f_{\mathrm{qnr}}-f_{\text {merge }}} \Theta\left(f-f_{\text {merge }}\right) \Theta\left(f_{\mathrm{qnr}}-f\right),
$$

where $\epsilon_{\mathrm{m}}$ is the fraction of mass energy radiated during merger phase which we assumed to be $\epsilon_{\mathrm{m}}=0.1, \Theta(x)$ is the step function, and $F(\mu / M)=(4 \mu / M)^{2}$. Finally, the energy spectrum in the ringdown phase is

$$
\begin{aligned}
\frac{\mathrm{d} E}{\mathrm{~d} f}= & \frac{A^{2} M^{2} f^{2}}{32 \pi^{3} \tau^{2}}\left\{\frac{1}{\left[\left(f-f_{\mathrm{qnr}}\right)^{2}+(2 \pi \tau)^{-2}\right]^{2}}\right. \\
& \left.+\frac{1}{\left[\left(f+f_{\mathrm{qnr}}\right)^{2}+(2 \pi \tau)^{-2}\right]^{2}}\right\} \\
\approx & \frac{1}{8} A^{2} Q M^{2} f_{\mathrm{qnr}} \delta\left(f-f_{\mathrm{qnr}}\right)[1+O(1 / Q)],
\end{aligned}
$$

where $Q=\pi \tau f_{\text {qnr }}$ is the quality factor, $\tau$ is the damping time of the quasi-normal mode of the newly formed black hole, and $A$ is the initial amplitude of the wave. The quality factor is approximately $Q \approx 12$, we assume that the initial amplitude $A=0.4$. The amplitude scales as $F(\mu / M)$ for non equal mass binaries.

The details of the calculation are shown in the Appendix. Here we present the final results in a form useful for future calculations. In the inspiral phase the signal to noise ratio is:

$$
\begin{aligned}
& \frac{S}{N}=\frac{M_{z}^{5 / 6}}{D_{100}} \sqrt{\frac{4 \mu}{M}} \\
& \times \begin{cases}0.95 \sqrt{1-3.26 \times 10^{-5} M_{z}^{10 / 3}} & \text { for } M_{z}<9.9 \\
1.21 \sqrt{1-0.197 M_{z}^{1 / 3}} & \text { for } 9.9<M_{z}<102 \\
0.00056 \sqrt{8.69 \times 10^{12} M_{z}^{-11 / 3}-1} & \text { for } 102<M_{z}<2027 \\
0 & \text { for } 2027<M_{z}\end{cases}
\end{aligned}
$$


where $M_{z}=M(1+z) / M_{\odot}$, and $D_{100}=D / 100 \mathrm{Mpc}$. In the merger phase the signal to noise ratio is:

$$
\begin{aligned}
& \frac{S}{N}=\frac{1}{D_{100}} \frac{4 \mu}{M}
\end{aligned}
$$

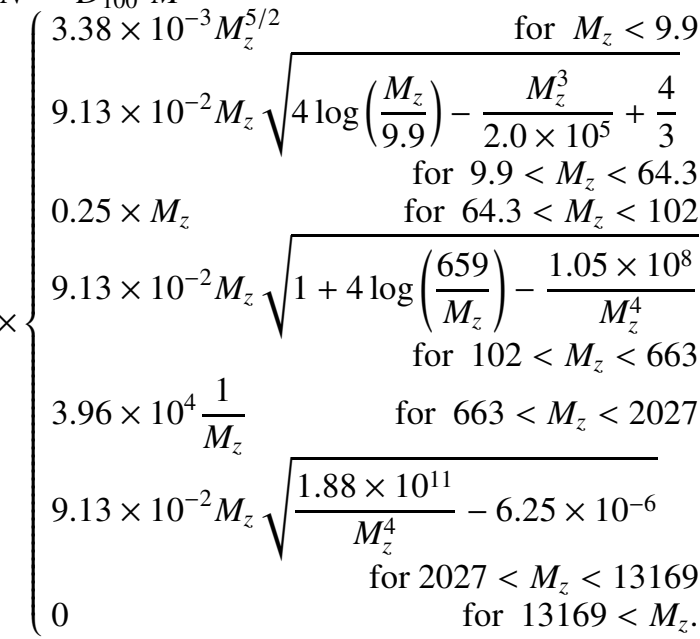

Finally in the ringdown phase the signal to noise is:

$$
\frac{S}{N}=\frac{1}{D_{100}} \frac{4 \mu}{M} \begin{cases}1.80 \times 10^{-4} M_{z}^{5 / 2} & \text { for } M_{z}<63.4 \\ 0.0938 M_{z} & \text { for } 63.4<M_{z}<650 \\ 4.126 \times 10^{4} \frac{1}{M_{z}} & \text { for } 650<M_{z}<13265 \\ 0 & \text { for } 13265<M_{z} .\end{cases}
$$

We have calculated the SNR using the above approximate formulae, as well as numerically integrating the noise spectrum given in Punturo (2004). The results are shown in Fig. 2. We also show for comparison the SNR for the initial LIGO configuration. The inspiral phase signal to noise decreases quickly with increasing mass when $(1+z) M>100 M_{\odot}$ for VIRGO. However, for VIRGO the merger and ringdown phase $\mathrm{S} / \mathrm{N}$ peaks at about 800-1000 $M_{\odot}$, where the LIGO sensitivity already drops down. Thus VIRGO may be able to catch the signal of black hole binary coalescence with the total masses above $500 M_{\odot}$. The sensitivity of VIRGO detector is similar to that of the initial LIGO for the merging binaries with the masses below $200 M_{\odot}$. However, the difference in sensitivity in the low frequency regime leads to improvement of the expected $S / N$ ratio in VIRGO for large redshifted masses.

\section{Detection rate}

In order to estimate the detection rate in VIRGO and LIGO we simulate the evolution of massive Population III stars, using the models of Belczynski et al. (2004). We made the same assumptions about the evolution of Population III stars: the initial mass function is assumed to have a slope of -2 above $100 M_{\odot}$ and extends up to $500 M_{\odot}$. We use the numerical results of Baraffe et al. (2001); Marigo et al. (2001); Heger \& Woosley (2002) to describe the stellar evolution tracks, and calculate the He core masses with the results of Heger \& Woosley (2002). The outcome of the nuclear evolution follows from Heger et al. (2003). We include pair instability supernovae so stars with initial masses between 140 and $260 M_{\odot}$ leave no remnants. Similarly to the population synthesis of Population I stars, the initial orbits are drawn from a distribution flat in logarithm, and the initial mass ratio distribution is flat. The binary fraction is assumed to be $10 \%$. For more details see Belczynski et al. (2004). This model of evolution of Population III binaries provides an estimate of the distribution of masses and lifetimes of the binaries. We assume a constant star formation rate between redshift 10 and 30, and obtain the black hole binary birth distribution function $F(M, t, z)=\Theta(z-10) \Theta(30-z) \sum \delta\left(M-M_{i}\right) \delta\left(t-t_{i}\right)$, where the sum goes over all the systems in the simulation. About $10^{-3}$ of the baryonic mass of the Universe must have been processed through Population III stars (Madau \& Rees 2001) which leads to the comoving rate of Population III star formation of $R_{\text {sfr }} \approx 1.4 \times 10^{-2} M_{\odot} \mathrm{Mpc}^{-3} \mathrm{yr}^{-1}$. The calculations are carried out for a flat cosmological model with $\Omega_{\mathrm{m}}=0.3, \Omega_{\Lambda}=0.7$ and the Hubble constant $H_{0}=67 \mathrm{~km} \mathrm{~s}^{-1} \mathrm{Mpc}^{-1}$. The luminosity distance, $D(z)$, is given by (see e.g. Bulik et al. 2004)

$D(z)=c(1+z) \int_{0}^{z}\left(1+z^{\prime}\right)\left|\frac{\mathrm{d} t}{\mathrm{~d} z^{\prime}}\right| \mathrm{d} z^{\prime}$,

where $t$ is the cosmic time:

$$
\left|\frac{\mathrm{d} t}{\mathrm{~d} z}\right|=\frac{1}{H_{0}(1+z) \sqrt{\Omega_{\mathrm{m}}(1+z)^{3}+\Omega_{\Lambda}}} .
$$

The observed black hole binaries coalescence rate is given by:

$\frac{\mathrm{d} R}{\mathrm{~d} M}=\int_{0}^{z_{\max }} \frac{\mathrm{d} f_{\text {coal }}(z)}{\mathrm{d} M} \frac{1}{1+z} \frac{\mathrm{d} V}{\mathrm{~d} z} \mathrm{~d} z$

where the maximum redshift $z_{\max }$ is estimated using Eqs. (A.2), (A.3) and (A.4) for the three phases of coalescence respectively, for a given SNR value in the detector. We have made a simplifying assumption that all events above a given $\mathrm{S} / \mathrm{N}$ are detected and that the detectors sensitivity does not depend on the direction to the source. The comoving volume element is:

$$
\frac{\mathrm{d} V}{\mathrm{~d} z}=\frac{4 \pi c D(z)^{2}}{1+z}\left|\frac{\mathrm{d} t}{\mathrm{~d} z}\right| .
$$

The black hole binary coalescence rate, $\mathrm{d} f_{\text {coal }}(z) / \mathrm{d} M$, depends on the birth rate of the binaries retarded by their evolutionary time:

$$
\frac{\mathrm{d} f_{\text {coal }}(z)}{\mathrm{d} M}=\int \mathrm{d} t^{\prime} F\left(M, t^{\prime}, z_{\mathrm{f}}\right),
$$

where $z_{\mathrm{f}}$ is the source formation redshift, which can be found by solving the following integral:

$t^{\prime}=\int_{z}^{z_{\mathrm{f}}}\left|\frac{\mathrm{d} t}{\mathrm{~d} z}\right| \mathrm{d} z$

In the calculation of the binary inspiral time we also consider the possibility that there are additional factors leading to tightening of the orbits. These may be due to interaction with the ISM or with stars in dense centers of galaxies, where such binaries are likely to sink. We model these processes by considering a model in which the orbits are additionally tightened (the binaries become harder) by a factor of ten.

We present the results of the rate calculation as a function of the signal to noise in Fig. 3. The Monte-Carlo calculation involved evolution of $10^{5}$ initial binaries, the redshift was discretized into the bins of the width $\mathrm{d} z=10^{-3}$. We have put the initial $10^{6}$ binaries into each redshift interval with the width $d z$ between $z=10$ and $z=30$, and found that the number of detected binaries with the $(S / N)>10$ was: 13951 in case of the 

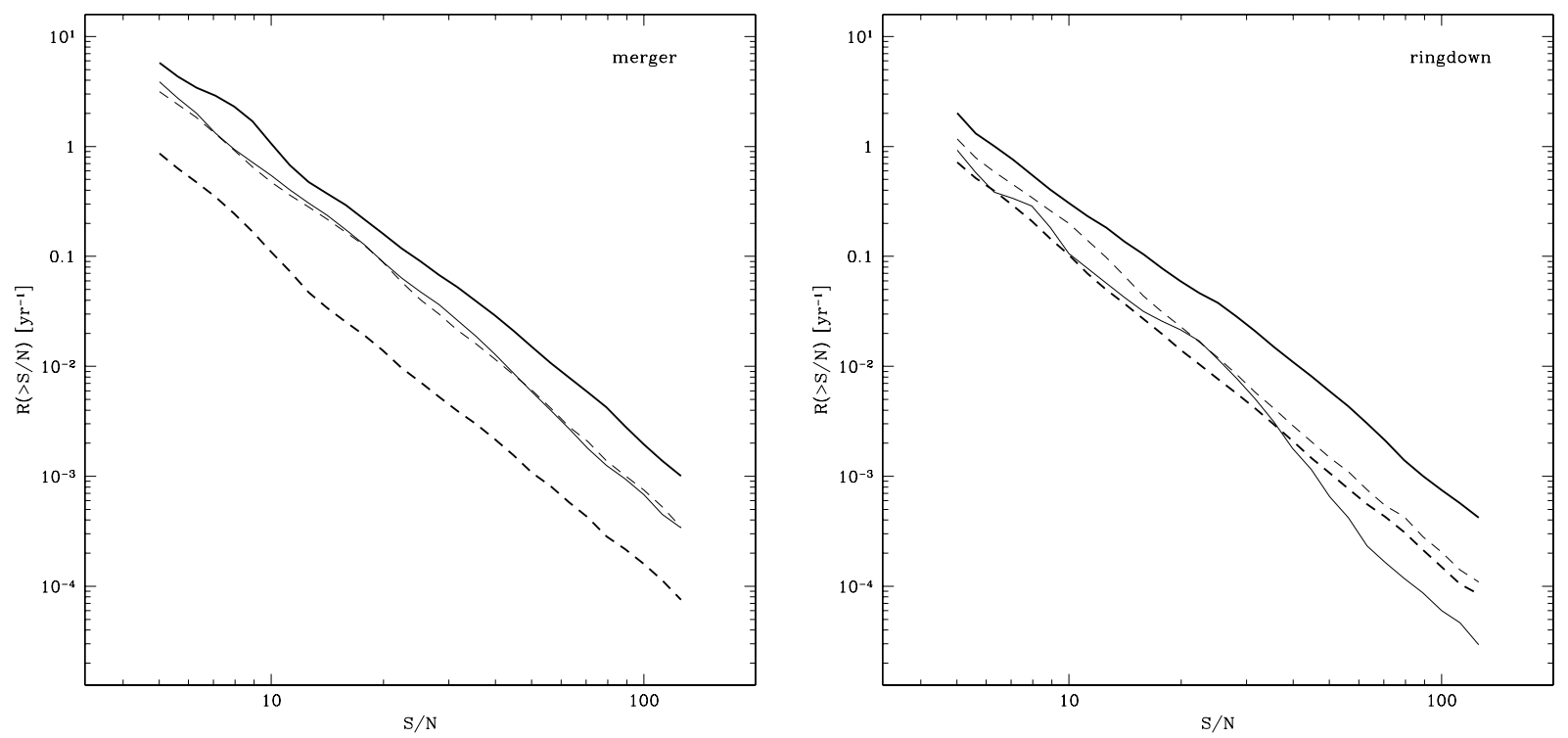

Fig. 3. Expected observed coalescence rate of Population III black hole binaries as a function of SNR for the VIRGO (solid lines) and initial LIGO (short dashed lines) detectors. The thick lines correspond to the case without hardening of the binaries while the thin lines show the case when all binaries are hardened by a factor of $\sim 10$. Left panel corresponds to the merger phase, and the right panel to the ringdown phase.

merger signal without hardening, and 16073 with hardening, and in the case of the ringdown signal 9396 without hardening and 8367 with hardening. Each plot contains two sets of curves: the rate for the VIRGO detector, the rate for the initial LIGO. These rates are lower by a factor about $\sim 10^{4}$ than the ones calculated for the advanced LIGO detector by Belczynski et al. (2004).

The detection rates do not depend strongly on the assumed evolution of binaries and are similar in the merger and the inspiral phase. For the assumed $S / N \approx 10$ the detection rate without hardening is $R(>S / N) \approx(1-10) \mathrm{yr}^{-1}$. It drops with increasing required $\mathrm{SNR}$ and for $S / N \approx 20$ it is about ten times less $\left(R(>S / N) \approx(0.1-1) \mathrm{yr}^{-1}\right)$. Since the required SNR in the detector corresponds roughly to the maximal distance out to which coalescences are detectable the rate drops as $(S / N)^{-3}$. On the other hand the rate scales as the inverse cube of the detector sensitivity - the minimum detectable strain amplitude. For the detection in the inspiral phase one ususally requires a $S / N$ cut of 8 , however for the case of merger and ringdown signal when the energetics and signal shape is not well known a higher $S / N$ ratio may be required.

In Fig. 4 we present the distribution of the redshifted total masses of the binaries detectable in merger and ringdown phases for the cases with and without hardening in the VIRGO detector. The distributions are normalized to unity. These distributions show the most probable masses of the coalescing binaries in the framework of the given model of evolution of black hole binaries: without hardening and with hardening by a factor of 10 . The shape of the distribution appears to depend strongly on the particular choice of the hardening. This can be understood in the framework of the discussion of various $\mathrm{BHBH}$ binary formation channels presented by Belczynski et al. (2004). However, we would like to stress that given the approximate model of the evolution of Population III binaries one can only conclude that the probable distribution of redshited total masses of the detectable binaries spans the range from $100 M_{\odot}$ to $700 M_{\odot}$, and the shape of the distribution varies strongly with the assumed model. Therefore the optimal search strategy would be to search the entire spectrum of the masses from $100 M_{\odot}$ to $1000 M_{\odot}$, as the current models can not provide more accurate predictions. The best strategy is to look for bursts of single frequency - signals from the ringdown phase.

\section{Summary}

We have calculated the signal to noise ratio from coalescing black hole binaries in VIRGO. The low frequency sensitivity of this detector leads to increased sensitivity to intermediate mass black hole mergers. We find that for the merger and ringdown signals VIRGO has a maximum of sensitivity for systems with the redshifted masses in the range from $500 M_{\odot}$ and $2000 M_{\odot}$. This makes VIRGO an especially valuable instrument in search for the signals from the intermediate mass black hole coalescences, as well as captures of stars by intermediate mass black holes in clusters.

Population III stars must have included a large number of high mass stars which should have left a significant number of black holes with masses above $100 M_{\odot}$. Some of them could have been formed binaries. Based on the evolutionary scenarios of such stars we calculate the properties of such binaries and estimate the rate with which they should be detectable by VIRGO. The rate is of the order of one detection per year for the design sensitivity of initial VIRGO and similar for the current LIGO sensitivity. While this number is not large by itself we note that it is comparable or even larger than the expected detection rate of the most often considered gravitational wave sources i.e. the double neutron star binaries (Belczynski et al. 2002; Kalogera et al. 2004). It should be noted that the number of the detectable coalescences of black hole binaries originating in Population III stars increases quickly with the detector sensitivity. For the advanced LIGO the expected rates are larger by a factor of $\sim 10^{4}$ (Belczynski et al. 2004). Therefore even modest improvements of the sensitivity will make the detection of these binaries more likely.

In calculating the rates we have made several simplifying assumptions that must be remembered. First, we have made use of the simplified formalism in treating the merger phase, namely we have assumed that the merger spectrum is flat. Moreover we have assumed that merger efficiency is $\epsilon_{\mathrm{m}}=0.1$. The main 
Merger, no hardening

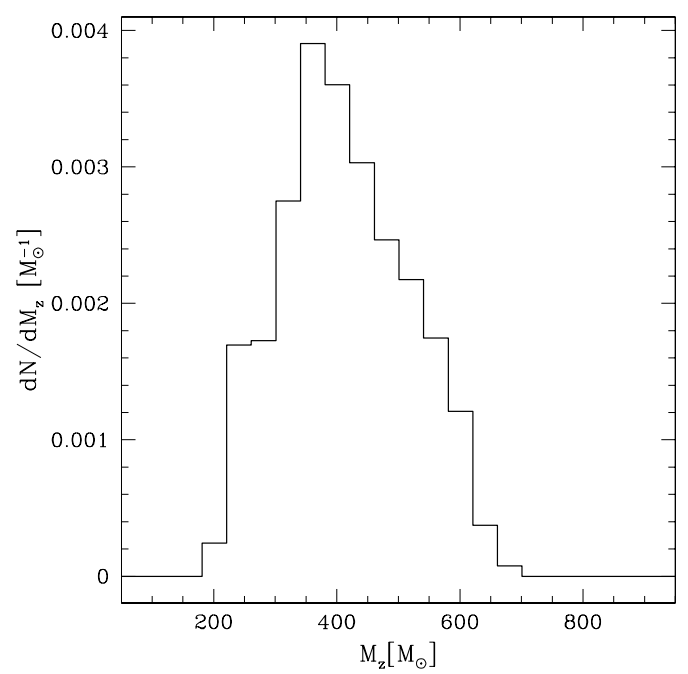

Ringdown, no hardening

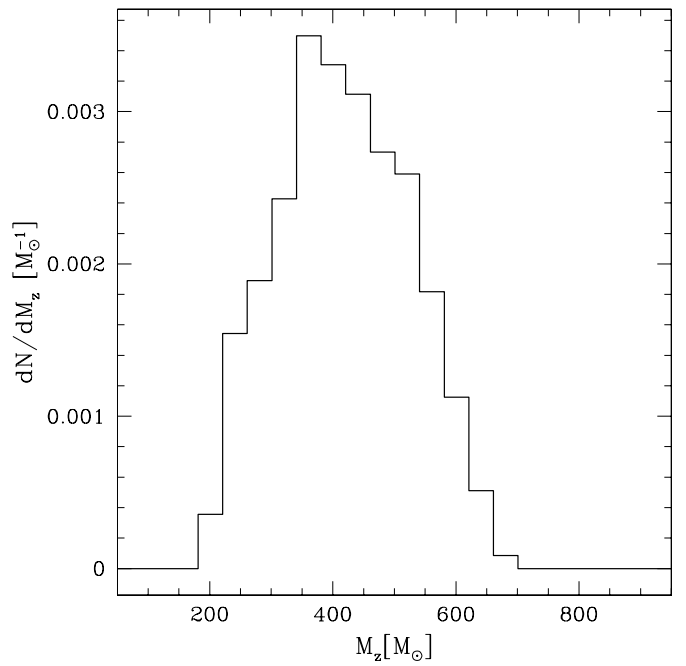

Merger, with hardening

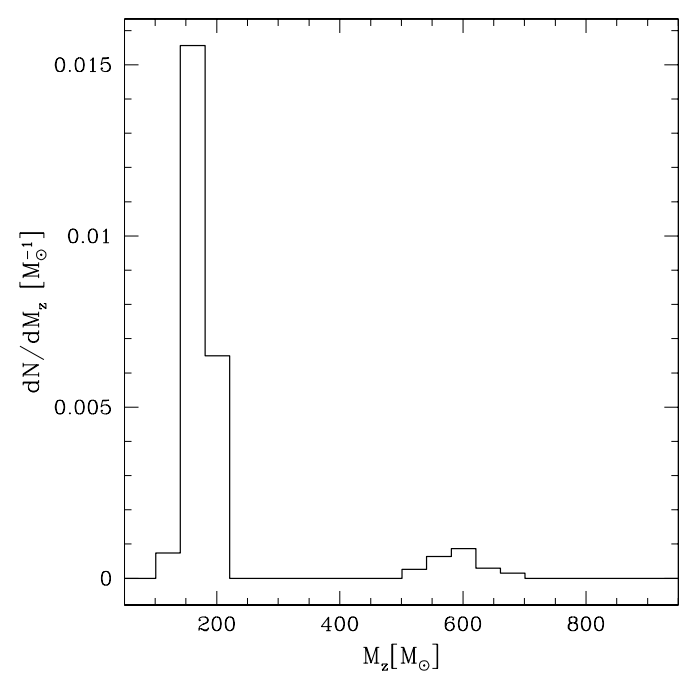

Ringdown, with hardening

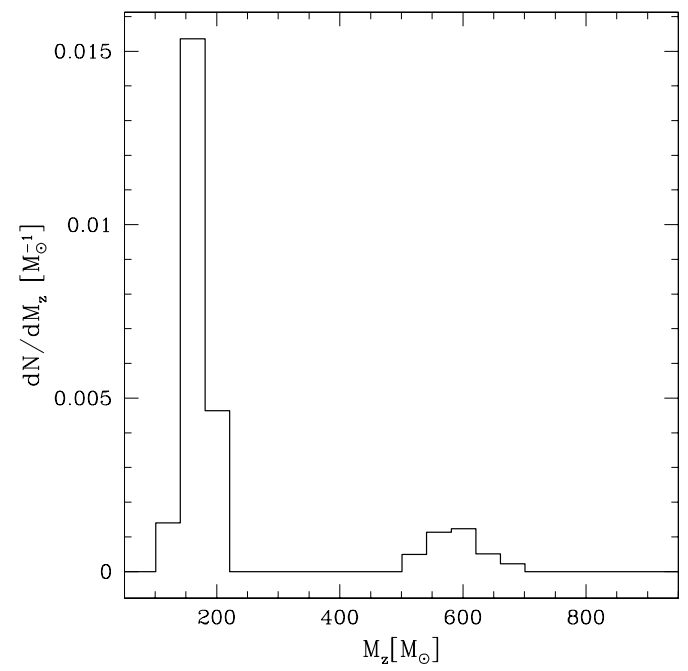

Fig. 4. Differential rate as a function of observed redshifted mass of black hole binary system. The top plots correspond to the detection with the merger signal, while the bottom ones to the ringdown case. The left panels show the case with no hardening of the binaries, while the right ones correspond to hardening of the orbits by a factor of 10. While the binaries were formed at redshifts from 10 to 30 , the detectable mergers happen at redshifts below unity. The distributions vary strongly with a particular model of Population III binary evolution. All plots are normalized to unity.

reason for this choice was consistency with the previous work of Flanagan \& Hughes (1998). The recent numerical work, e.g. for the case of double neutron star mergers, shows that the energy in the merger phase may be smaller (Shibata \& Taniguchi 2006). The numerical calculations of the double black hole mergers (Baker et al. 2006; Campanelli et al. 2006b) demonstrate that the merger efficiency is $\approx 3 \%$. In calculations of the merger of spinning black holes the efficiency can reach $\approx 6 \%$ (Campanelli et al. 2006a). We note, however, that the $S / N$ ratio scales only as $\sqrt{\epsilon_{\mathrm{m}}}$ and therefore the results weakly depend on the assumed value of this parameter. Therefore the value $\epsilon_{\mathrm{m}}=0.1$ that we use may lead to overestimation of the rate by a factor of less than 2 . When treating the ringdown phase we have assumed that the initial amplitude is $A=0.4$, following Flanagan \& Hughes (1998). The signal to noise ratio scales linearly with $A$, however one can easily see the impact of a different choice of $A$ on the predicted rates from Fig. 3.
Similarily to our previous work (Bulik et al. 2004; Belczynski et al. 2004) the rates have been calculated assuming that the detector is omnidirectional and that all sources have a larger $S / N$ than a given value. In a detailed calculations one has to take into account all these effects to better estimate the rate, like Nutzman et al. (2004) have done in the case of Population I double neutron star binaries.

One should be still cautious when dealing with estimates of the properties of the Population III stars. The rate we calculate depends on the actual shape of the initial mass function of Population III stars, and on their binary fraction. The knowledge of evolution of Population III stars is only known from numerical models, and hence the models evolution of Population III binaries are only approximate. However, these uncertainties and the possible role that Population III stars could have played in the early Universe makes it even more interesting to look for traces of their existence, and gravitational waves searches provide such an opportunity. 
Thus there is a number of factors that may influence the value of the expected rates presented in this paper. First, the energy scale and the waveforms in the merger phase, and the amplitude of the ringdown phase are not known as precisely as the ones for the inspiral phase. In order to account for this one may have to require a higher $S / N$ than in the case of inspiral. Second, there is a number of unknowns in the formation and evolution of Population III stars. However, a plausible set of parameters describing the problem yields the rates that are quite interesting for the existing interferometric detectors. Therefore we consider that it is very important to include the ringdown and merger templates in the search for merging binaries, and to look for signals from merging binaries across the entire sensitivity range of the interferometric gravitational wave detectors.

Acknowledgements. This work was supported by the KBN grant 1P03D002228.

\section{References}

Abramovici, A., Althouse, W. E., Drever, R. W. P., et al. 1992, Science, 256, 325 Baker, J. G., Centrella, J., Choi, D.-I., Koppitz, M., \& van Meter, J. 2006, Phys. Rev. D, 73, 104002

Baraffe, I., Heger, A., \& Woosley, S. E. 2001, ApJ, 550, 890

Belczynski, K., Kalogera, V., \& Bulik, T. 2002, ApJ, 572, 407

Belczynski, K., Bulik, T., \& Rudak, B. 2004, ApJ, 608, L45

Bradaschia, C. 1990, Nucl. Instrum. Methods Phys. Res., A289, 518

Bromm, V., Coppi, P. S., \& Larson, R. B. 1999, ApJ, 527, L5

Bromm, V., Coppi, P. S., \& Larson, R. B. 2002, ApJ, 564, 23

Bulik, T., Belczyński, K., \& Rudak, B. 2004, A\&A, 415, 407

Campanelli, M., Lousto, C. O., \& Zlochower, Y. 2006a, ArXiv General Relativity and Quantum Cosmology e-prints

Campanelli, M., Lousto, C. O., \& Zlochower, Y. 2006b, Phys. Rev. D, 73, 061501

Flanagan, É. É., \& Hughes, S. A. 1998, Phys. Rev. D, 57, 4535

Heger, A., \& Woosley, S. E. 2002, ApJ, 567, 532

Heger, A., Fryer, C. L., Woosley, S. E., Langer, N., \& Hartmann, D. H. 2003, ApJ, 591, 288

Kalogera, V., Kim, C., Lorimer, D. R., et al. 2004, ApJ, 601, L179

Madau, P., \& Rees, M. J. 2001, ApJ, 551, L27

Marigo, P., Girardi, L., Chiosi, C., \& Wood, P. R. 2001, A\&A, 371, 152

Nutzman, P., Kalogera, V., Finn, L. S., Hendrickson, C., \& Belczynski, K. 2004, ApJ, 612, 364

Punturo, M. 2004, VIRGO internal note VIR-NOT-PER-1390-51

Saigo, K., Matsumoto, T., \& Umemura, M. 2004, ApJ, 615, L65

Shibata, M., \& Taniguchi, K. 2006, Phys. Rev. D, 73, 064027

Wyithe, J. S. B., \& Loeb, A. 2004, ApJ, 612, 597

\section{Appendix A: General formulae for the signal to noise ratio}

In order to find the general formulae for the signal to noise ratio we insert Eqs. (4)-(6) and (2) into Eq. (1) and perform the integration. We introduce a dimensionless variable

$v=\frac{(1+z) \alpha f_{\mathrm{m}}}{f_{\text {merge }}}$.
For the inspiral phase we obtain:

$$
\frac{S}{N}=\left\{\begin{array}{rr}
\sqrt{\mathcal{F}_{\mathrm{i}}\left[12 \alpha^{1 / 3}-\frac{99}{10} \alpha^{-1 / 3}-\frac{11}{10} \alpha^{-1 / 3} v^{10 / 3}-\alpha^{4}\left(\frac{f_{\mathrm{s}}}{f_{\mathrm{m}}}\right)^{11 / 3}\right]} & \text { for } v<1 \\
\sqrt{\mathcal{F}_{\mathrm{i}}\left[12 \alpha^{1 / 3}-11\left(\frac{v}{\alpha}\right)^{1 / 3}-\alpha^{4}\left(\frac{f_{\mathrm{s}}}{f_{\mathrm{m}}}\right)^{11 / 3}\right]} & \text { for } 1<v<\alpha^{2}(\mathrm{~A} .2) \\
\sqrt{\mathcal{F}_{\mathrm{i}}\left[\alpha^{1 / 3}\left(\frac{\alpha^{2}}{v}\right)^{11 / 3}-\alpha^{4}\left(\frac{f_{\mathrm{s}}}{f_{\mathrm{m}}}\right)^{11 / 3}\right]} & \text { for } \alpha^{2}<v<\alpha f_{\mathrm{m}} / f_{\mathrm{s}} \\
0 & \text { for } \alpha f_{\mathrm{m}} / f_{\mathrm{s}}<v
\end{array}\right.
$$

where

$\mathcal{F}_{\mathrm{i}}=\frac{2[(1+z) M]^{5 / 3}[4 \mu / M]}{55 \pi^{4 / 3} D(z)^{2} h_{\mathrm{m}}^{2} f_{\mathrm{m}}^{1 / 3}}$.

For the merger phase we obtain:

$$
\frac{S}{N}=\left\{\begin{array}{lll}
\sqrt{\mathcal{F}_{\mathrm{m}}\left[\frac{4}{3} v^{3} \frac{\kappa^{3}-1}{\kappa^{3}}\right]} & \text { for } \quad v<1 \\
\sqrt{\mathcal{F}_{\mathrm{m}}\left[4 \ln v-\frac{4}{3}\left(\frac{v^{3}-\kappa^{3}}{\kappa^{3}}\right)\right]} & \text { for } 1<v<\kappa \\
\sqrt{\mathcal{F}_{\mathrm{m}}[4 \ln \kappa]} & \text { for } \quad \kappa<v<\alpha^{2} \\
\sqrt{\mathcal{F}_{\mathrm{m}}\left[1+4 \ln \left(\frac{\kappa \alpha^{2}}{v}\right)-\frac{\alpha^{8}}{v^{4}}\right]} & \text { for } & \alpha^{2}<v<\kappa \alpha^{2} \\
\sqrt{\mathcal{F}_{\mathrm{m}}\left[\alpha^{8} v^{-4}\left(\kappa^{4}-1\right)\right]} & \text { for } & \kappa \alpha^{2}<v<\alpha f_{\mathrm{m}} / f_{\mathrm{s}} \\
\sqrt{\mathcal{F}_{\mathrm{m}}\left[\left(\frac{\kappa \alpha^{2}}{v}\right)^{4}-\left(\frac{\alpha f_{\mathrm{s}}}{f_{\mathrm{m}}}\right)^{4}\right]} & \text { for } \alpha f_{\mathrm{m}} / f_{\mathrm{s}}<v<\kappa \alpha f_{\mathrm{m}} / f_{\mathrm{s}} \\
0 & \text { for } & \kappa \alpha f_{\mathrm{m}} / f_{\mathrm{s}}<v
\end{array}\right.
$$

where

$\kappa=f_{\text {qnr }} / f_{\text {merge }}$

and

$\mathcal{F}_{\mathrm{m}}=\frac{\epsilon_{\mathrm{m}} M(1+z)^{2}(4 \mu / M)^{2}}{10 \pi^{2} D(z)^{2} h_{\mathrm{m}}^{2} f_{\text {merge }}(\kappa-1)}$.

The fraction of the mass emitted in gravitational waves during the merger phase has been assumed to be $\epsilon_{\mathrm{m}}=0.1$ (Flanagan $\&$ Hughes 1998).

For the ringdown phase the result is

$$
\frac{S}{N}=\sqrt{\frac{(1+z)^{3} M^{2} A^{2} Q(4 \mu / M)^{2}}{20 \pi^{2} D(z)^{2} f_{\mathrm{qnr}} S_{h}\left[f_{\mathrm{qnr}} /(1+z)\right]}} .
$$

Note that the signal to noise in the merger phase scales as $\epsilon_{\mathrm{m}}^{1 / 2}$, and in the ringdown phase it scales linearly with the initial amplitude $A$. 